\title{
Reflets
}

Revue d'intervention sociale et communautaire

\section{Problématisation des dimensions psychiques et sociales dans l'intervention, une perspective socioclinique}

\section{Marie-Chantal Doucet}

Volume 17, numéro 1, printemps 2011

Psychologisation de l'intervention sociale : enjeux et perspectives

URI : https://id.erudit.org/iderudit/1005236ar

DOI : https://doi.org/10.7202/1005236ar

Aller au sommaire du numéro

Éditeur(s)

Reflets, Revue d'intervention sociale et communautaire

ISSN

1203-4576 (imprimé)

1712-8498 (numérique)

Découvrir la revue

Citer cet article

Doucet, M.-C. (2011). Problématisation des dimensions psychiques et sociales dans l'intervention, une perspective socioclinique. Reflets, 17(1), 150-174.

https://doi.org/10.7202/1005236ar
Résumé de l'article

À partir d'une perspective socioclinique, ce texte s'emploie à dégager des pistes de réflexion sur la nécessaire problématisation des dimensions psychiques et sociales dans l'intervention. Celle-ci sera définie comme espace théorique, mais aussi espace d'un rapport à l'autre où s'articulent les aspects affectifs, cognitifs et sociaux. Sera examinée la portée théorique du récit sur soi en tant que production de connaissances qui conjuguent ces aspects. Il serait réducteur de penser que le travail sur soi ne serait qu'une réponse psychologisante à l’impératif de production de l'individu.
Tous droits réservés @ Reflets, Revue d'intervention sociale et communautaire, 2011
Cecument est protégé par la loi sur le droit d'auteur. L'utilisation des services d’Érudit (y compris la reproduction) est assujettie à sa politique d'utilisation que vous pouvez consulter en ligne.

https://apropos.erudit.org/fr/usagers/politique-dutilisation/ 


\section{Problématisation des dimensions psychiques et sociales dans l'intervention, une perspective socioclinique}

Marie-Chantal Doucet, Ph. D. (sociologie)

Professeure, Faculté des sciences humaines

École de travail social, Université du Québec à Montréal

"Mais il est de la plus haute importance méthodologique, et quasi décisif pour les principes des sciences humaines en général, qu'en traitant scientifiquement des faits psychiques, on ne fait pas nécessairement de la psychologie. »

Georg Simmel

\section{Résumé}

À partir d'une perspective socioclinique, ce texte s'emploie à dégager des pistes de réflexion sur la nécessaire problématisation des dimensions psychiques et sociales dans l'intervention. Celleci sera définie comme espace théorique, mais aussi espace d'un rapport à l'autre où s'articulent les aspects affectifs, cognitifs et sociaux. Sera examinée la portée théorique du récit sur soi en tant que production de connaissances qui conjuguent ces aspects. Il serait réducteur de penser que le travail sur soi ne serait qu'une réponse psychologisante à l'impératif de production de l'individu. 
Mots clés : Individu, société, connaissance, clinique, intervention

\section{Abstract}

From a socio-clinical, this text seeks to identify avenues for reflection on the necessary articulation of social and psychological dimensions in the intervention. This will be defined as theoretical space but also within one over the other aspects, which revolve emotional, cognitive and social. Will discuss the theoretical significance of the narrative self as a knowledge production that combines these aspects. It would be simplistic to think that the work itself is merely a response to the imperative psychologising production of the individual.

Key words: Individual, society, knowledge, clinical, intervention

\section{Introduction}

"Les sciences sociales ont eu tendance à rejeter les questions qui se rapportent à l'individu en considérant qu'elles sont du domaine de la psychologie. "
Les sciences sociales ont eu tendance à rejeter les questions qui se rapportent à l'individu en considérant qu'elles sont du domaine de la psychologie. Pourquoi opposer aussi simplement l'individuel au social? Le problème n'est pas nouveau et on peut affirmer qu'il est à l'origine même de la sociologie. Déjà, Durkheim avait reproché son psychologisme à Simmel, son contemporain. Dès la formation de cette discipline apparait donc le conflit individu-société/psychologie-sociologie. Pour Durkheim, il n'y a de sentiment signifiant - pour la science - que collectif tandis que Simmel affirme que les " faits sociaux» - expression de Durkheim - sont aussi des faits psychiques. En 1959, dans son ouvrage Morale théorique et science des mours, Gurvitch avait relevé une erreur commune au rationalisme, à l'empirisme et au criticisme qui consistait dans le rejet de l'expérience sensible en tant qu'objet de connaissance sociologique. D'un côté, la théorie du choix rationnel met en scène un sujet libre et responsable 
"La réflexion sur les problèmes sociaux et l'intervention ne peut contourner la question de l'individu social."

"...qu'il est nécessaire de reconnaître la subjectivité en tant que composante de la connaissance. » et de l'autre, les analyses critiques et structuralistes posent que l'individu serait produit par les structures et les discours. Le reste appartiendrait à la psychologie.

Dépositaire pragmatique des sciences humaines, au carrefour de l'individuel et du social, l'intervention sociale ne peut cependant éluder une remise en cause approfondie, si possible libérée de prétention disciplinaire, des modes d'appréhension de l'individu sans perdre tout sens de ce qu'est intervenir auprès de quelqu'un. L'intervention sera ici définie comme cet espace entre le praticien et le sujet, espace de construction théorique qui est aussi espace d'un rapport à l'autre. La réflexion sur les problèmes sociaux et l'intervention ne peut contourner la question de l'individu social. Cette perspective ne doit cependant pas être définie comme une psychologie ni même une microsociologie, mais comme un élargissement de la théorie sociale qui tiendra compte du caractère réflexif des conduites et de la pluralité des styles de vie. La réflexion sur l'intervention doit éviter de se cantonner dans les limites des méthodologies de l'intervention collective, individuelle et groupale comme si elles étaient opposées, reproduisant de manière un peu grossière la fameuse dichotomie individuel/social présente aux premières heures des sciences sociales. Enfin, cette réflexion ne devrait pas être une affaire de méthode, mais interpelle plutôt le champ de la connaissance et celui de l'action dont les connexions sont, il faut bien le dire, parfois ténues dans le monde de l'intervention sociale. La clinique en sciences humaines, que l'on peut considérer dans son sens large - individuel et collectif - comme modalité d'un rapport sujet/objet se caractérise par la nécessité constante de lier connaissance et intervention dans un mouvement circulaire. Elle nous enseigne aussi qu'il est nécessaire de reconnaitre la subjectivité en tant que composante de la connaissance. Sera considéré comme clinique ce qui appréhende le sujet — individuel, familial, communautaire - à travers un dispositif dont le centre est le rapport sujet/objet en tant que relation sociale où les protagonistes sont impliqués, que ce soit dans un travail visant l'évolution, le développement, la transformation ou encore la production de connaissances. C'est à partir de cette démarche qu'est soutenue l'idée de l'impossibilité logique d'une rupture entre les dimensions 
psychiques et sociales. Le problème social est généralement lisible à travers une approche sociostructurelle qui correspond à la figure abstraite de l'individu. L'on tiendra compte de deux opérations toujours liées : la saisie d'un phénomène qui sort de l'ordinaire et un jugement de valeur qui suggère la mise en place de correctifs. Il s'agira de planifier un processus d'intervention à partir d'une procédure dont la caractéristique est la reproduction d'un modèle (Dumont, 1984). Pourtant, l'accentuation de la forme impersonnelle et objectivée de l'individu conduit à l'apparition d'une deuxième figure correspondant à une distanciation croissante face aux rôles et au raffinement de la connaissance subjective. L'appareillage théorique qui sert à analyser les problèmes sociaux échoue à saisir les significations des conduites relevant de cette connaissance. Le point de vue sociostructurel ne rend pas compte de la pluralité des styles de vie et du caractère réflexif des conduites humaines. Les phénomènes semblent envisagés seulement dans leur extériorité structurelle. La connaissance subjective mérite d'être problématisée de façon plus claire en intégrant les dimensions psychiques et sociales afin de mieux définir ce qu'est une intervention. Cet article s'emploie à contribuer à la réflexion sur ce sujet. Trois titres divisent et coiffent notre étude :

1. L'élargissement du champ de la connaissance;

2. Le récit sur soi comme production épistémique;

3. Pour une perspective socioclinique de l'individu.

\section{L'élargissement du champ de la connaissance}

L'espace de pensée de la connaissance en sciences humaines et sociales s'était constitué autour de la scientificité et de sa déclinaison en disciplines au XIX ${ }^{\mathrm{e}}$ siècle. Pourtant, des auteurs de cette même époque ne tarderont pas à élargir le champ de la connaissance en intégrant d'autres savoirs. La psychanalyse, par exemple, s'est formée autour de l'idée d'une connaissance inconsciente et, à sa manière, elle a remis en cause le récit d'une science dont le projet serait la maîtrise du réel. 
L'épistémologie contemporaine, notamment par la voie de la sociologie de la connaissance, permet de reconsidérer le domaine en intégrant encore d'autres savoirs - ordinaires, pratiques, spirituels, littéraires, subjectifs, etc. - comme autant de facultés de l'esprit humain susceptibles d'être analysés en tant que productions individuelles-sociales. Dès lors, se développe une socio-anthropologie de la connaissance (Farrugia, 2002; Schurmans, 2010) intégrant l'objet "subjectif " et conduisant à une reconsidération de la rupture philosophique entre objectivité et subjectivité. Dans l'intervention, elle peut se traduire par une certaine opposition entre l'affectif et le cognitif. L'identification du champ de l'affectivité a depuis longtemps été attribuée à la psychologie alors qu'à l'intérieur même de cette discipline, ce fameux débat cognitif/affectif demeure fondamental; et l'on peut affirmer qu'aujourd'hui, le domaine cognitivo-comportemental est dominant.

Les thérapies cognitivo-comportementales connaissent trois vagues (Cottraux et Dattilio, 2007) :

1. Le comportement (1930-1980);

2. La cognition (1970-1990);

3. L'émotion (de 1990 à nos jours).

Loin de s'exclure, ces trois moments correspondent à trois notions qui s'emboîtent les unes aux autres. Dans la période comportementale, tout ce qui se rapporte au domaine motivationnel y est exclu. Le noyau logique est causal; le comportement y est défini comme " un enchaînement ordonné d'actions destinées à adapter l'individu à une situation telle qu'il l'interprète " (Cottraux, 2007, p. 4). L'objet de cette théorie à ses premières démarches est l'étude du comportement observable. Elle se fonde sur une recherche expérimentale et objectiviste, étudiant les relations entre stimuli et réponse. Le comportement humain s'élabore progressivement au travers d'apprentissages successifs, des associations simples entre stimuli et réponses, acquises dans l'enfance servant de matériaux de base aux comportements plus complexes de la vie adulte. La deuxième vague concerne la cognition en tant qu'acte de connaissance : comment l'humain acquiert, organise, utilise le savoir, comment l'humain traite l'information en définitive. On 
s'intéresse ici à la perception, à la pensée, à l'action. Datant d'une quinzaine d'années, la troisième période introduit l'émotion au milieu de la mentalité cognitiviste, prolongeant ainsi le débat soulevé par Darwin (1992), puis Ekman (1994) entre les partisans de l'universalisme biologique et les relativistes pour qui les émotions sont avant tout culturelles (Mead,1973). Chez les tenants de la théorie cognitivo-comportementale, le comportement est le résultat adaptatif de schémas cognitifs qui déterminent les émotions (Beck, 1983). La cognition ici est donc causale.

\section{Événement $\rightarrow$ Évaluation cognitive $\rightarrow$ Émotion}

Par ailleurs, le domaine socioaffectif a déjà été causal. Par exemple, les problèmes scolaires étaient réunis sous d'autres vocables que ceux employés aujourd'hui - TDAH, handicaps, etc. Au cours des années 1970, il est question de mésadaptation socioaffective; le sujet étant socialement inadapté, il requiert des mesures de resocialisation. Dans ce cas, l'émotion produite par cette inadaptation conduit aux échecs cognitifs. Tout se passe comme si le sujet est entièrement déterminé par les structures et qu'il suffit d'adapter l'individu au système. Dans le premier cas - cognitivo-comportemental - on peut observer que l'individu, par ses choix qui doivent être rationnels, détermine le social tandis qu'ici, le social détermine l'individu. Dans ces deux registres explicatifs qui semblent opposés, le problème se définit à la base dans l'ordre d'une causalité plutôt linéaire.

Il est donc permis de penser qu'il s'agit d'un problème beaucoup plus vaste qu'une dispute disciplinaire ou interdisciplinaire, engageant la réflexion sur d'autres pistes qui seraient plutôt transdisciplinaires. Il s'agirait de se demander si le domaine de l'affectivité ne serait pas toujours " [...] l'émanation d'un

"S'occuper de l'affectivité ne revient pas simplement à individualiser ou à psychologiser les choses. » milieu humain donné et d'un univers de sens et de valeurs " (Lebreton, 2007, p. 333) et d'une certaine manière, une forme de connaissance socialement construite. S'occuper de l'affectivité ne revient pas simplement à individualiser ou à psychologiser les choses. Au contraire, les émotions et sentiments sont relatifs aux cadres sociohistoriques tout comme ils dépendent des contextes psychosociaux (Farrugia, 2002). Les connaissances produites à 
"L'objet

d'intervention, aussi

objet de connaissance, sera non seulement déterminé par les conditions objectives, mais aussi construit par le sujet qui l'observe. " leur sujet s'inscrivent aussi bien dans les domaines politique, éthique, sociologique et psychologique. Elles concernent plusieurs logiques croisées tels les savoirs théoriques et les savoirs d'action, les techniques et le sens commun. La connaissance doit être comprise en tant que configuration plutôt qu'un ensemble de structures disciplinaires refermées sur elles-mêmes. Sa forme est en effet mouvante et s'appréhende sur du continu plutôt que sur les dichotomies. Les expériences intérieures sont à reconsidérer aussi en tenant compte de la contingence et de la différenciation individuelle, ce qui en fait des objets complexes. Ce qui relève de l'affectuel aurait plutôt tendance à échapper aux sciences en général ainsi qu'à la concurrence théorique et disciplinaire. Ce sera au contraire dans les imbrications complémentaires qu'émergeront des savoirs implicites qui incluent la connaissance subjective, aux sources de l'activité sociale. Celle-ci est en effet constituée à partir de structures sociales objectives, mais aussi d'un monde doté d'affects.

Les cadres de la connaissance - Gurvitch - ne sont pas qu'externes, structurels et formels, c'est-à-dire qu'ils ne relèvent pas seulement d'enjeux de pouvoir; de rapports politiques et de conditions économiques, mais ils sont aussi sociosymboliques, culturels et implicites. Penser en ces termes fait appel à un constructivisme de la connaissance (Farrugia, 2002). Ce changement de paradigme met en lien de réciprocité les intelligences subjective et objective, les dynamiques psychiques et les contextes sociaux. L'objet d'intervention, aussi objet de connaissance, sera non seulement déterminé par les conditions objectives, mais aussi construit par le sujet qui l'observe. Dès lors, il faut prendre en compte la relation observateur/observé. L'évolution de cette dyade à travers l'intervention est redevable à l'intersubjectivité et à la créativité, ce qui affirme le rôle central des sujets connaissant qui se situe tant du côté de l'observateur que de l'observé. Abbott (2006) rappelle aussi que la connaissance nait tout autant d'accommodements et de conflits que de paradigmes et de codification.

Au moment où il écrit son ouvrage sur la théorie des émotions au début des années 1930, alors que l'on voit poindre des travaux 
ouverts au courant comportemental, Vygotsky constate que ce qui prime est l'émotion. Cette séparation entre l'aspect intellectuel et l'aspect affectif sera au centre de ses préoccupations, car il s'agit d'un problème qui traverse l'ensemble des sciences humaines. Par ailleurs, Vygotsky porte un regard critique sur l'approche neurologique des émotions et met en doute qu'un ensemble de neurones dessinent les cartes cognitives. Il introduit la nécessité de considérer le contexte social dans l'explication : les autres interviennent aussi dans la vie des individus, ce qui dépasse l'explication des phénomènes uniquement à partir des schèmes cognitifs. Le rapport avec le monde environnant s'avère complexe. Les émotions et sentiments ne se réduisent pas à des déterminismes biologiques ni à des schémas intellectuels. Le sentiment est exprimé par le langage. Celui-ci s'insère dans une culture, c'està-dire dans une interactivité fondamentale. Il faut donc interrelier affect-cognition-social. Selon Vygotsky (1998), « [1]'examen de l'interaction des processus mentaux intersubjectifs peut éclairer à sa manière l'activité des processus mentaux intrasubjectifs" (p.15). Il s'agit plus largement de saisir la signification des liens s'établissant entre le sujet individuel et les "globalités sociohistoriques ». C'est ainsi que l'intervention auprès d'un seul individu est une mise en relation avec la société globale. L'interprétation se fait au bénéfice d'une transformation sociale et individuelle et non strictement dans le seul but de révéler l'inconscient ou des structurants culturels inconscients. En ce sens, Vygotsky se situe au carrefour d'une herméneutique et d'une actancialité. Proche de la pensée de Dilthey (1947) sur l'esprit humain, il se situe dans le registre de la compréhension. Cette posture a souvent été attribuée à un certain psychologisme. Par exemple, Dilthey a pu dans ses premiers travaux accorder plus d'importance à la conscience individuelle (Apel, 2000). Il ne tarde pas à constater que cette psyché ne peut évoluer séparément de la société. Dilthey affirme que chaque récit et chaque œuvre d'art ne sauraient être compréhensibles sans une communauté reliant celui qui parle et celui qui comprend. L'individu pense et agit dans une communauté; la compréhension n'est possible que dans une telle sphère de communauté. 
"La pensée a toujours un destinataire; cette pensée est médiatisée par le langage et la culture."
Le cognitif, l'affectif et le social se trouvent liés de manière conflictuelle ou harmonieuse dans l'action. À la différence de Piaget (2005) pour qui le sujet trouve en lui les ressources pour agir sur le monde - les stades de développement de l'épistémologie génétique -,Vigotsky avance que le social fournit également des ressources. Il s'oppose au cognitivisme expérimental pour proposer plutôt une théorie de l'action située, c'est-à-dire en contexte. Le sujet y crée en faisant des compromis entre les contraintes et le symbolique - l'" imaginaire d'autorité " et l'" imaginaire moteur » selon les termes d'Enriquez (1992). L'activité est toujours située dans un contexte particulier. L'art du sujet sera sa capacité à changer de registre selon les situations. La pensée a toujours un destinataire; cette pensée est médiatisée par le langage et la culture. Mais il ne s'agit pas seulement d'une application déterministe du dehors au dedans; il s'agit aussi d'une recréation du dehors par le dedans.

Or, de son côté, la posture critique répond de manière réductive à la question de la psychologisation de l'intervention. Il ne suffit pas de reléguer tout le domaine de l'affectif en psychologie qui, à l'heure actuelle, tente de l'évacuer en le rapportant à la dominante cognitive. Nous avons vu que les questions affectuelles posent problème pour toutes les disciplines, y compris le travail social. Par ailleurs, la thèse critique en travail social repose sur de trop nombreuses simplifications. Elle pose que le travail social devrait obligatoirement se trouver du côté du collectif et des structures, tandis que les questions d'ordre individuel et affectif sont par nature psychologisantes et par conséquent du côté du pouvoir. Elle s'appuie, entre autres, sur la pensée de Castel et collab. (1981) sur la psychologisation du travail social qui, sur un fond de discours moralisateur, refuse à ce dernier toute indépendance d'esprit face à la psychologie : "Il faut le reconnaître, dit-il, c'est la psychologie et la psychopathologie qui ont donné son nouveau code d'interprétation et ses nouvelles technologies d'intervention à la profession du travail social » (p. 61). Il s'agit d'un texte qui ne possède aucune assise empirique propre à relever un tel discours chez les travailleurs sociaux. Au contraire, dans Le déclin des institutions, qui s'appuie sur des entretiens avec les «professionnels 
de l'aide à autrui ", Dubet (2002) fait part du malaise qui habite les travailleurs sociaux à la recherche d'un sens quant à leurs actions. La question du sens demeure fondamentale chez les intervenants sociaux (Renaud, 2001). Une recherche en cours sur les savoirs implicites des intervenants sociaux fait effectivement ressortir le problème de la perte de sens qui engage bien plus à débattre autour de la gestionarisation de l'intervention face à la souffrance quotidienne et d'une organisation du travail qui, sous couvert d'interdisciplinarité, découpe les tâches. On parle ici d'une souffrance liée aux enjeux sociaux de l'intervention sociale, d'une souffrance dite sociale qui concerne d'ailleurs aussi bien les clientèles des services sociaux que les intervenants eux-mêmes, quoique d'une manière différente. Or, comprendre et interpréter la souffrance n'est pas une activité strictement psychologique. L'étude de la souffrance ouvre plutôt sur une réflexion complexe reposant sur une articulation de l'individuel et du social. La question de la psychologisation de l'intervention sociale resurgit; on doit se préoccuper de sa pertinence dans le débat actuel. Ne serait-il pas possible de dépasser cette thèse un peu réductive qui se développe d'après l'axe pouvoir/contrepouvoir? Les modèles critiques en travail social posent un schéma a priori, celui du pouvoir des structures sur l'individu. Pourtant dans l'intervention, ce qui se trouve en premier ce n'est pas l'ordre social, mais comment s'articule le mouvement entre les contraintes de cet ordre et les actions quotidiennes.

La perspective critique demeure une nécessité. Elle gagnerait toutefois à intégrer les réflexions contemporaines sur un individu qui cherche à devenir «le sujet de son histoire » (de Gaulejac, 2009) et qui par ses actions contribue aux transformations du social. Le processus d'individuation des sociétés contemporaines amène une ambiance épistémique où tous les courants théoriques sur le comportement humain en psychologie comme en sociologie cherchent à développer une théorie de la pensée qui met au centre l'individu en lien avec son environnement. La psychanalyse contemporaine dégage une troisième topique dont les instances seraient le soi et l'objet. Ici, le dedans et le dehors ne se trouvent pas dans un conflit structural, mais plutôt dans un rapport 
"...le travail sur soi doit être envisagé comme une activité sociale..." d'interaction (Reid, 2008). On pourrait tout aussi bien avancer l'idée d'une sociologisation de la psychologie. C'est pourquoi la question semble devenue plus complexe. La réflexion est encore à faire sur une possible articulation du psychologique et du social. Les discussions présentes semblent pour le moment plus enclines à établir les périmètres des disciplines professionnelles, ce qui constitue un terrain peu propice aux échanges.

Or, aux déterminismes traditionnels, celui des structures sur l'individu et celui de l'individu sur la société, devrait s'ajouter pour un réel élargissement de la connaissance en sciences sociales une compréhension selon laquelle le travail sur soi serait une expérience épistémique où s'articulent le cognitif, l'affectif et le social. Toute démarche de connaissance devant être considérée comme une activité sociale, le travail sur soi doit être envisagé comme une activité sociale qui, dans le contexte d'individuation des sociétés actuelles, se trouve au fondement de la modernité contemporaine. La réflexivité générerait à travers le travail sur soi des propriétés transformatrices non seulement de l'individu, mais aussi de la société. Le récit représente l'une des formes privilégiées de ce travail de l'individu sur lui-même.

\section{Le récit sur soi comme production épistémique}

Une dualité dans l'appréhension des problèmes humains distingue le modèle et le récit. Le modèle sera plus près de ce qui est habituellement associé à la recherche scientifique. Il s'agit de structures de pensée qui amènent l'observateur à voir les phénomènes à partir d'un schéma précis. Pourtant, deux limites importantes peuvent être repérées dans le modèle : a) la première est que le comportement sera observé de façon distanciée en le rapportant à un cadre de référence. De la sorte, l'observation laisse échapper le sens au profit de la description des faits. Par exemple, les modèles diagnostics en psychiatrie basent uniquement leurs critères sur les données qui s'insèrent dans la catégorisation de critères diagnostics; b) la seconde limite se situe sur le plan de 
l'analyse. Cette dernière demeure descriptive. Elle tend même à se confondre au raisonnement ordinaire et remet peu en cause le contexte social où se produit le raisonnement. Par exemple, dans l'établissement du diagnostic du trouble déficitaire de l'attention, on soumet une grille questionnaire aux parents et aux enseignants avec des critères qui laissent largement place à l'interprétation sans se préoccuper des enjeux psychiques et sociaux dans lesquels les évaluateurs sont eux-mêmes impliqués : «L'enfant oublie souvent de faire ses devoirs "; "Souvent se laisse facilement distraire par des stimulus sans importance "; "A souvent du mal à soutenir son attention dans les travaux et dans les jeux » (American Psychiatric Association, 1994). Les questionnaires sont construits sur la base de présuppositions communes à une communauté savante qui elle-même a peu de distance avec le sens commun.

Si le modèle a encore la faveur, l'intervention comprise comme cadre d'un rapport entre deux sujets qui cherchent ne peut se constituer sans l'existence d'un récit : récit de souffrance, d'exclusion ou de malaise existentiel. Le récit se caractérise par sa polysémie, car l'auteur, tout en étant une personne singulière, s'est individué dans une culture donnée. Les savoirs produits, qui relèvent d'une intelligence subjective, sont portés à un premier niveau de compréhension par les individus. Le récit individuel doit être pourtant considéré comme un savoir individuel-social. C'est ce que démontre la sociologie clinique, notamment avec Sévigny (2009) et ses recherches en Chine auprès de patients psychiatriques. La narration de $\mathrm{Lu} \mathrm{Lu}$ sur sa "carrière psychiatrique " dans la Chine contemporaine recèle d'histoire sociale et fait bien ressortir l'ambiance culturelle d'une Chine à la recherche d'elle-même. Ce qui sera au centre de l'analyse sera précisément l'intervention en santé mentale dans une Chine en pleine transformation, vécue de l'intérieur par Lu Lu. Pour Sévigny, l'individu ne peut donner sens à son expérience sans référer au moins implicitement au social. À travers la narration de $\mathrm{Lu} \mathrm{Lu}$, qui exprime une expérience chargée d'émotions et de sentiments divers, on peut observer d'importantes transformations dans la pratique psychiatrique qui passe de la rééducation à la prise en compte de la qualité de vie tout en suivant le courant biomédical de l'Occident, ce qui donne 
"Le récit peut être considéré comme une forme de connaissance à la fois témoin et constitutive de la transformation sociale. » d'excellentes indications sur les changements sociaux opérés dans la Chine actuelle. La notion d'épreuve (Martuccelli, 2006) renvoie à une conception de l'individu comme sujet et comme acteur qui doit composer avec des situations. Il ne s'agit pas seulement d'une patiente schizophrène subissant sa maladie, mais de $\mathrm{Lu} \mathrm{Lu}$, schizophrène, travaillée par l'épreuve, mais également activement impliquée dans "la reconstruction de soi ", notamment dans l'activité même de se raconter. Le récit peut être considéré comme une forme de connaissance à la fois témoin et constitutive de la transformation sociale.

\section{L'individuation}

"Dans l'intervention, l'humain n'est pas qu'un objet empirique, mais un sujet qui agit sur sa vie."
Comment donc entendre ces récits? Tout dépend de ce que l'observateur cherche. Dans une relation d'intervention, celui-ci devrait s'efforcer de lier structures et expérience individuelle dans sa compréhension de l'épreuve. Dans l'intervention, l'humain n'est pas qu'un objet empirique, mais un sujet qui agit sur sa vie. Cette individuation n'est pas à confondre avec l'individualisme comme idéologie faisant croire à un sujet " expert de sa vie » et entièrement responsable de ses choix. Sur le plan de l'épistémologie des sciences sociales, il faut faire la distinction entre l'individualisme méthodologique et une sociologie de l'individuation. Le premier fait ressortir un individu abstrait, évoquant la seule explication et en fin de compte la seule réponse individuelle à l'épreuve. Peu de questions portent sur les structures pourtant dominantes et plus largement sur la part sociale des souffrances (Renault, 2009). Une sociologie de l'individuation telle que définie par Martuccelli considère le processus d'individuation comme principe premier des sociétés contemporaines. En ce sens, l'individuation peut être considérée comme une macrosociologie (Martuccelli, 2009). Ces années-ci, le thème majeur de la souffrance témoigne d'une nervosité collective (Ehrenberg, 1998); or des récits individuels, il est possible de tirer les relations implicites et parfois même tout à fait explicites entre les problèmes sociaux et la vie intérieure. 
C'est ce lien qui fera l'objet d'une sociologie de l'individuation. Comment l'individu résiste, crée, joue le jeu social et comment en parle-t-il?

Par ailleurs, il faut aussi remettre en question les déterminismes. Même si elle se targue parfois d'utiliser le narratif souvent naïvement expliqué, l'intervention contemporaine conduit à inscrire le récit dans un certain ordre linéaire en accord avec l'impératif de causalité. Les deux ordres de causalité repérés par Bergson (1979) lorsqu'il critique le positivisme se trouvent effectivement ancrés dans l'intervention classique et nous pourrions ici prendre le risque de les lier de façon un peu large à plusieurs postures adoptées par cette dernière. Il s'agit de la " causalité poussée " que nous pourrions faire correspondre à la prévention et de la "causalité attraction " qui touche la remémoration. Considérons le problème suivant qui pourrait impliquer ces deux ordres de causalité :

\section{Causalité poussée (prévention)}

$$
\begin{aligned}
& \mathrm{A}=\text { Cause (père alcoolique) } \\
& \text { donc } \\
& \mathrm{B}=\text { Effet (fils alcoolique) }
\end{aligned}
$$

Dans ce premier cas, une vision concentrique de la société que l'on retrouvera notamment dans l'écologie sociale dont découle l'approche écosystémique laisse voir un individu dépersonnalisé, résultat d'une équation simplifiée des facteurs de risque et de protection. Nous ne reprendrons pas ici les définitions des écosystèmes - onto, micro, méso, exo, macro et enfin chronosystème. Il suffit de retenir qu'ils sont tous considérés comme ayant une incidence sur l'individu dont l'action semble préprogrammée. D'autre part, les interventions semblent ne porter que sur l'ajustement de l'individu à ces systèmes. Paradoxalement, on fera d'ailleurs de l'individu l'expert de sa vie. La tendance sera d'envisager les comportements selon des séquences redevables à ce qui les précède et à induire aussi que tel comportement conduit à telle conséquence. Chaque séquence est un effet de la précédente et produit la suivante. Le même raisonnement 
"...l'excès de cohérence peut aussi laisser échapper une variété infinie de possibles. " s'applique concernant la prévention. Celle-ci s'appuie sur la logique d'intervenir sur ce qui n'a pas eu lieu, comme si l'agir humain était à ce point prévisible. Le temps humain sera ainsi composé au futur antérieur : tel enfant qui frappe les autres à quatre ans l'aura échappé belle; grâce à l'intervention préventive, il aura évité la délinquance. En effet, on pose que les actions peuvent tenir tout entières dans la finalité des schèmes explicatifs qui en fait les reconstituent comme si elles étaient entièrement prévisibles.

\section{Causalité attraction (remémoration)}

$$
\begin{aligned}
& \mathrm{A}=\text { Effet (fils alcoolique) } \\
& \text { à cause de } \\
& \mathrm{B}=\text { Cause (père alcoolique) }
\end{aligned}
$$

Dans ce schème classique, la remémoration sera le fil conducteur du récit. L'un des effets thérapeutiques du récit en psychologie ou en sociologie clinique sera l'effet de cohérence. À travers le récit de son histoire, le sujet construit sa vie, attribuant par exemple, son alcoolisme à celui du père et la trame du récit sera cette relation vécue en tant qu'épreuve. Cette dernière est ici considérée comme cause ultime de toute une série d'expériences douloureuses. Mais l'excès de cohérence peut aussi laisser échapper une variété infinie de possibles. La théorie du trauma qui s'inscrit dans ce schème d'intervention est d'ailleurs de plus en plus remise en question en psychanalyse (Reid, 2008).

Le récit, qui tente de façon bien humaine de reconstruire un soi fragilisé, ne devrait pas se dérouler finalement au sein d'un modèle explicatif qui chercherait la formule génératrice des conduites. Au milieu de la pluralité des styles de vie et des événements, il est pour le moins douteux de conclure que tel effet aura toujours sa cause dans le passé ou encore que tel facteur engendrera un risque pour le futur. Le temps ainsi envisagé, écrit Bergson (1979, p. 11), "n'est qu'un espace idéal où l'on suppose alignés tous les événements passés, présents et futurs ».

Par ailleurs, on peut opposer le fait que la tendance est à "l'ordre narratif». Pour le dire plus simplement, ne dirait-on pas que tout le monde aujourd'hui dit " faire du narratif ». Or 
faire du narratif ne s'improvise pas. Cela doit aussi être considéré comme le fruit d'un travail personnel dans lequel l'intervenant non seulement s'éprouve lui-même, c'est-à-dire subjectivement, mais également s'interroge sur son rôle d'observateur en considérant que son intervention consiste d'abord en un "acte de voir " (Doucet, 2009). L'acte de voir est à insérer dans le contexte social de sa production. Ce n'est pas tout de faire du narratif. Il importe de connaître le filet théorique à partir duquel le praticien recueillera le récit. On assiste aujourd'hui à certaines dérives à ce sujet. Le témoignage, largement utilisé dans les colloques afin d'appuyer la réussite de telle pratique d'intervention médicale, psychologique ou sociale, conduit à produire un individu qui a conquis sa maladie par la force de sa volonté et s'inscrit clairement dans les thèses dominantes de l'individu responsable. Dans ce cas, le récit se transforme en un modèle porteur d'une double signification. D'une part, le modèle se caractérise par sa fonction validante sur le plan de la connaissance (Gardin, 2001). Il est en effet difficile d'avoir une confirmation de la théorie qui soit aussi précise que dans la modélisation. Un récit peut ainsi servir d'appui au modèle par une déconstruction où les segments narratifs sont rangés sous des catégories posées a priori. D'autre part, un récit peut se voir promu au rang de modèle moral, cette fois au sens héroïque du terme, c'est-à-dire en tant qu'histoire exemplaire où le héros s'est rétabli grâce à son courage et à sa détermination.

Le cadre épistémologique présentant l'intégration de la vie sociale dans un mécanisme objectif qui serait ancré dans l'idée de discontinuités - ou de ruptures - a jusqu'ici dominé les approches et construit les objets de recherche. Cependant, ce modèle est aujourd'hui de plus en plus inefficace, car il laisse échapper un monde en mouvement, toujours en continuité et qui ne cesse de se redéfinir lui-même. Développée d'une façon extrêmement intéressante par Martuccelli (2006), la notion d'épreuve ne se définit pas en termes de rupture ou de discontinuité; elle engage à faire une mise à distance avec la temporalité narrative, permettant une ouverture sur d'autres interprétations possibles qui mettent en relation continue les processus sociohistoriques et l'existence quotidienne des acteurs. 
"Dans le contexte d'individuation des sociétés contemporaines, les récits sur soi sont à la fois révélateurs de la dimension symbolique du social et dotés d'une fonction sociohistorique."
C'est pourquoi les récits ne peuvent davantage être enfermés dans les limites de l'une ou de l'autre des théories du comportement humain qui sont autant de configurations sociales de l'individu. Ce point de vue ouvre plutôt la voie à un pluralisme pragmatique (Doucet, 2009).

La notion d'épreuve enfin propose de voir les contextes sociaux non pas simplement comme déterminant, mais comme défis. Quelles sont les marges de jeux du sujet devant les multiples épreuves individuelles-sociales du monde actuel? Les humains ne sont pas des automates qui subiraient le système. Cette perspective ouvre sur de multiples potentialités de l'intervention. Plutôt que de se désoler sur les institutions qui enferment les sujets aussi bien que les discours, comme les analyses foucaldiennes nous ont habitués à le penser, serait-il possible de tendre l'oreille vers d'autres récits qui racontent quelque chose ayant à voir avec une subjectivation? Dans le contexte d'individuation des sociétés contemporaines, les récits sur soi sont à la fois révélateurs de la dimension symbolique du social et dotés d'une fonction sociohistorique. Ce n'est pas pour cela que sera nié l'impact des contraintes structurelles. Il ne s'agit pas de nier le caractère normatif de la vie sociale. Par conséquent, il n'est pas question de rejeter une analyse dialectique des forces en présence. La question est bien : suffit-elle pour comprendre l'action humaine? Les individus ne peuvent être considérés simplement comme les résultats de conditions d'existence et l'activité psychique n'est certainement pas la reproduction pure et simple de ces conditions. Les postures critiques qui ne se situent qu'à l'extérieur donnent à penser que l'action des individus se fait uniquement en résonnance aux prescriptions structurelles. L'écoute des récits, c'est-à-dire une posture qui serait cette fois intérieure à la relation clinique, permet de penser que le raisonnement et les affects ne se trouvent pas à être l'exacte traduction de ce qui est prescrit de penser et de ressentir.

La connaissance subjective, heureusement, ne se restreint pas à la seule souffrance, mais aussi aux habiletés créatives. L'apparition de l'individu à l'avant-scène de la conscience contemporaine introduit un rapport particulier avec le monde social dont une sociologie de la connaissance subjective pourrait rendre compte. 
"Le processus d'individuation amène une solitude devenue peu à peu l'un des sentiments cruciaux de l'époque."
Aussi, Farrugia (2010, p. 3) avance que le narrateur « doit se comprendre comme un concentrateur de flux cognitifs et sensitifs, un topo focalisateur de signes à déchiffrer, un lieu de passage de significations circulantes dans un univers de significations sociales croisées ". Le récit, pourrait-on dire, produit l'individu tout autant que ce dernier produit le récit. Or, l'action qu'est le travail sur soi ne peut être conçue comme étant déterminée mécaniquement. Les sujets pensent leur vie et la racontent. Cette action tend à transformer le contexte. Le travail sur soi sera traversé des tensions entre l'individuel et le social. Une recherche récente sur les pratiques du travail sur soi (Doucet, 2010) permet de conclure que ces pratiques diversifiées, telles que psychothérapies, ateliers psychospirituels ou groupes de soutien, se situent dans le registre de la réflexivité en tant que modalité contemporaine des rapports sociaux. Le processus d'individuation amène une solitude devenue peu à peu l'un des sentiments cruciaux de l'époque. Nous n'avons pas l'espace nécessaire pour nous pencher davantage cette question qui est développée ailleurs (Doucet, 2007). Cependant, il est nécessaire de comprendre la solitude comme un objet complexe qui ne devrait pas être analysé uniquement comme la conséquence d'une modernité narcissique ou comme une émotion de désolation, mais comme empreint de tensions paradoxales entre un quant-à-soi réflexif et la société. La réflexivité sur sa vie devient le moyen terme entre soi et autrui. Dans la modernité contemporaine, elle agit comme véhicule de la dimension émancipatoire de la connaissance qui répond au besoin que l'on pourrait dire inscrit dans l'ontologie humaine de se connaitre en tant qu'humanité (Apel, 2000). Il serait réducteur de penser que le travail sur soi n'est qu'une réponse psychologisante à l'impératif de production de l'individu.

Le travail sur soi sera traversé des tensions entre l'individuel et le social. Ce sera au moyen d'un travail sur soi réflexif que ces tensions jamais vraiment résolues seront remises en question. $\mathrm{La}$ réflexivité en effet se trouve au cœur des pratiques du travail sur soi. Elle peut ainsi être vue comme un élément essentiel de ce passage historique entre un «nous » indifférencié et un «je » qui émerge. Nous avons ailleurs avancé que la solitude en tant qu'expérience 
sociale représente un passage vers ce qui constitue le fondement de la modernité contemporaine, c'est-à-dire la reconnaissance d'une tension paradoxale entre un quant-à-soi réflexif et la société (Doucet, 2007). Le paradoxe, écrivions-nous, «tient au fait que cet espace qui semble au plus haut point privé définit un rapport particulier avec la société; le quant-à-soi se conçoit comme hors du monde alors qu'il en est l'un des fondements " (p.168). C'est de cette intériorité comme conscience de soi, mais aussi comme relation à l'altérité, dont il est question dans le travail sur soi. La réflexivité sur sa vie devient le moyen terme entre soi et autrui.

\section{Pour une perspective socioclinique de l'individu}

Mieux que quiconque, Simmel a su faire ressortir le caractère fluctuant de la vie sociale et la nécessité d'une lecture mettant en relation complémentaire l'extériorité et l'intériorité. Pourtant contemporain de Freud, il ne s'est pas directement exprimé sur l'inconscient psychanalytique. En revanche, son épistémologie s'attarde au psychisme. Les processus politiques, religieux et économiques sont redevables aux processus psychiques qui euxmêmes se trouvent imprégnés des actions sociales. Essentiellement, Simmel pose les actions réciproques comme notion centrale pouvant expliquer la socialisation. Celle-ci ne sera pas comprise dans le sens qu'on lui confère habituellement, c'est-à-dire comme interiorisation des normes, mais bien comme processus ontologique de liaison. Ce qui se manifeste chez les individus sous forme de mobilité psychique, de récit, de silence, de conflits, constitue en fait le contenu ou la matière de la socialisation. L'approche structurelle ne peut expliquer qu'un nombre limité de phénomènes. Lorsqu'il est affirmé que les relations de pouvoir se retrouvent dans toute relation humaine, tout n'est pas dit. C'est oublier le processus complexe du croisement des cercles sociaux dans lesquels interagissent les individus au quotidien. La multiplication de ces cercles dans la modernité contemporaine — travail, famille, amour, loisirs, etc. — dont les formes different 
"La socialisation se fera donc avec l'individuation." selon les histoires conduit à une plus grande différenciation des individus et à une plus grande distance au rôle. Nous aurons recours à Martuccelli (2006) quand il dit :

À une société homogène, peu différenciée, avec peu de cercles sociaux, correspond un acteur faiblement singularisé et soumis à la tradition, tandis qu'une société complexe, hautement différenciée et rationalisée, produit un individu se déployant dans une pluralité de cercles sociaux, croisant un nombre chaque fois plus élevé d'inconnus, soumis à diverses stimulations nerveuses et destiné à accomplir plusieurs tâches différentes. (p. 9)

C'est en raison des différences individuelles qu'existe une possibilité de relation. L'absence de différence, c'est-à-dire une identification complète, conduirait plutôt à la fusion. La socialisation se fera donc avec l'individuation. C'est peut-être justement dans la foulée de l'individualisme naissant qu'apparaissent à la fin du XIX ${ }^{\mathrm{e}}$ siècle les discours sur la société. Ce sera paradoxalement cette différenciation qui générera la conscience du lien social, une solidité non pas statique, mais fluctuante. "La manière dont les relations réciproques s'assemblent et se divisent, voilà par quoi il faut commencer ", écrit Simmel (1999, p.57).

D'après cette perspective qui s'intéresse à l'individu et au lien social, il est possible de faire intervenir une conception qui pourrait nous amener à travailler à l'articulation des dimensions psychiques et sociales dans l'intervention et ainsi à la saisir d'une manière différente. La catégorie du lien social ne cherche pas qu'à saisir les mécanismes de la contrainte structurelle sur l'individu, mais à comprendre ce qui constitue l'être social d'un individu. Pour Farrugia (2008), le lien social dérive d'une spontanéité sociale qui serait nécessaire et pourtant contingente toujours à faire et donc toujours à penser. Les actions n'ont de sens qu'à l'intérieur des liens. C'est pourquoi l'intervention n'a de sens qu'à l'intérieur d'une conception dialogique où les acteurs ne se situent pas seulement dans une expérience psychologique, mais 
aussi sociale et cognitive puisqu'il leur faut déchiffrer aussi un ensemble de signes. Opposer le social et le psychologique freine la pensée sur l'intervention alors que ces deux aspects se combinent nécessairement dans cette action. Il est donc vain de chercher à les opposer puisque "les deux nous sont donnés " (Dubet, 2005). Il serait beaucoup plus fécond d'œuvrer pour une articulation de ces dimensions. Nous avons vu que le récit sur soi autour d'une épreuve permet d'introduire une configuration de l'individu d'une manière différente, mettant en lien les dimensions affectuelles, cognitives et sociales qui se combinent de manière harmonieuse ou conflictuelle selon les contextes. Le modèle, tel que nous l'avons défini, qu'il se range sous la catégorie fonctionnelle ou critique, fait de l'individu un agent abstrait, déterminé par un modèle social qui lui préexiste et le produit. Une perspective socioclinique nous enseigne plutôt que l'individu n'est pas un produit fini et déterminé par les modèles, mais qu'il est plutôt un processus. Il composera tout au long de son existence avec les épreuves personnelles et les enjeux sociaux.

Selon Dubet (2005), c'est parce que l'humanité s'explique à la fois par ses individus et ses sociétés que le problème de cette opposition obsède les sciences humaines. Ce problème est d'autant plus obsédant que l'individu ressort de la masse dans la modernité contemporaine. Le sujet se trouverait entre déterminisme et autonomie (Dubet, 2005). À travers ses pratiques, il devient générateur de nouvelles formes sociales. Le récit sur soi présente l'intérêt d'interroger l'articulation du rapport individusociété en reconnaissant l'expérience des sujets comme forme de connaissance. Il faut donc l'appréhender comme catégorie cognitive de la construction des liens sociaux. Cette connaissance s'inscrit en effet dans les liens d'altérité quotidiens qui sont objets de réflexion chez les sujets. La démarche du praticien est double : il cherche à comprendre le sens de la communication et il cherche une signification de second niveau à ce qu'il entend. Il ne peut voir le monde qu'en ayant recours à la connaissance qu'il en a. À cet égard, il n'existe pas de différence de nature entre connaissances savante, pratique et ordinaire. La connaissance savante obéit au principe de la double herméneutique (Giddens, 1984) puisqu'elle 
a affaire à un monde déjà constitué d'interprétations. Son rôle sera d'interpréter ces interprétations à partir de ses propres schèmes. De même, le travail de l'intervenant est essentiellement un travail d'interprétation. La clinique s'appuie donc sur une analyse de contenu du langage en tant que configuration d'un savoir individuel-social. Cette conception s'oppose au déterminisme et à ses conséquences méthodologiques où le sujet sera appréhendé du point de vue des grands ensembles - genre, milieu social, etc. - en dépit de la singularité des histoires. Il ne s'agit pas de rejeter toute étude référant à ces "méthodologies de l'intervention ", mais de les problématiser autrement en revoyant le catalogue des approches traditionnelles à la lumière des transformations sociales et des développements théoriques. L'individu est en travail. À la fois cognitif, affectif et social, il serait erroné de ne le considérer qu'à travers sa position dans les structures.

\section{Conclusion : L'intervention comme complexe individuel-social}

C'est à cet individu fortement singularisé et différencié que s'adressera l'intervention. Partant de là, la constitution de la connaissance en savoirs disciplinaires perd de l'acuité. La socialisation est un phénomène psychique et les phénomènes psychiques se construisent dans la socialisation. Ce sera précisément avec ce changement de perspective que l'intégration des dimensions psychiques et sociales dans l'intervention paraît n'être que psychologisation. Pourtant, nous avons vu que les problèmes sociaux auxquels s'attaquent les intervenants ne sont pas qu'extérieurs et les individus ne sont pas que les dépositaires des discours. Les sujets pensent eux aussi. La tendance sera de laisser de côté cette connaissance. L'autre sera perçu comme généralisé. Des catégories sont construites - les exclus, les marginaux, les psychotiques - alors que la seule saisie extérieure des phénomènes rend impossible une compréhension intégrant la diversité des histoires. Les positions sociales ne sont pas suffisantes 
pour expliquer les conduites. L'individu compose entre ses appartenances aux grands ensembles - genre, classe sociale, etc. et ses liens quotidiens. L'observation devrait porter sur le contenu et ses configurations et non strictement sur les processus sociaux des grands ensembles. De même, la seule lecture des processus psychologiques dont l'étude par la voie de l'expérimentation domine actuellement en psychologie demeure elle aussi insuffisante. D'ailleurs, cette dernière discipline fait actuellement tout en son pouvoir pour évacuer la question subjective.

On voit bien que le fait de se pencher sur la subjectivité individuelle n'induit pas forcément que les questionnements sont de nature strictement psychologique. L'objet des sciences sociales, et a fortiori de l'intervention, ne consiste pas seulement dans le travail structurel, mais aussi dans l'observation des conduites réflexives. Par ailleurs, ceux qui réfléchissent à l'intervention doivent prendre en compte l'élargissement du champ de la connaissance dans lequel on s'interroge de plus en plus sur d'autres regroupements possibles sur le plan des savoirs.

\section{Bibliographie}

ABBOTT, Andrew (2006). "Le chaos des disciplines ", dans Jean Boutier, Jean-Claude Passeron et Jacques Revel (dir.), Qu'est-ce qu'une discipline?, Paris, Éditions EHESS, 260 p.

AMERICAN PSYCHIATRIC ASSOCIATION (1994). Manuel diagnostique et statistique des troubles mentaux, (DSM-IV), $4^{\mathrm{e}}$ édition, Washington, DC, American Psychiatric Publishing, 943 p.

APEL, Karl-Otto (2000). La controverse expliquer-comprendre, Paris, Éditions du Cerf, 384 p.

BECK, Aaron, et collab. (1983). Cognitive therapy of dépression, New York, The Gilford Press, 425 p.

BER GSON, Henri (1979). La pensée et le mouvant : essais et conférences, Paris, PUF, 612 p.

BRESSON, Maryse (dir.) (2006). La psychologisation de l'intervention sociale : mythes et réalités, L'Harmattan, 269 p.

CASTEL, Robert, Françoise CASTEL et Anne LOVELL (1981). La société psychiatrique avancée, le modèle américain, Paris, Grasset, 365 p.

CHARMILLOT, Maryvonne, et collab. (2008). Émotions et sentiments : une construction sociale, approches théoriques et rapports au terrain, Paris, L'Harmattan, $212 \mathrm{p}$.

COTTRAUX, Jean, et collab. (2007). Thérapies cognitives et émotions : la troisième vague, Issy-lesMoulineaux, Masson, 205 p.

DARWIN, Charles (1992). L'origine des espèces au moyen de la sélection naturelle ou la préservation des races favorisées dans la lutte pour la vie, Flammarion, Paris, 604 p. 
DE GAULEJAC,Vincent (2009). Qui est « je »? Sociologie clinique du sujet, Paris, Seuil, 218 p.

DE GAULEJAC,Vincent, Fabienne HANIQUE et Pierre ROCHE (dir.) (2007). La sociologie clinique, Enjeux théoriques et méthodologiques, Erès, 347 p.

DE SINGLY, François, et Danilo MARTUCCELLI (2009). Les sociologies de l'individu, Paris, A. Colin, $128 \mathrm{p}$.

DILTHEY, Wilheim (1947). Le monde de l'esprit (Die geistige Welt), traduction M. Rémy, 2 volumes, Paris, Aubier-Montaigne.

DOUCET, Marie-Chantal (2007). Solitude et sociétés contemporaines, une sociologie clinique de l'individu et du rapport à l'autre, Presse de l'Université du Québec, 179 p.

DOUCET, Marie-Chantal (2009). "Théories du comportement humain et configurations sociales de l'individu ", Sociologie et sociétés, Vol. 41, № 1, Presse de l'Université de Montréal, p. 35-53.

DOUCET, Marie-Chantal (2010). «Travailler sur soi par le récit, une sociologie de la connaissance subjective ", dans Christina Constantopoulou (dir.), Récits et temps sociaux, L'Harmattan, (à paraître).

DUBET, François (2005). "Pour une conception dialogique de l'individu », réf. du 17 février 2011, EspaceTemps.net, Textuel, 21.06.2005, http//espacestemps.net/document 1438.html.

DUBET, François (2002). Le déclin des institutions, Paris, Seuil, 421 p.

DUMONT, Fernand (1984). «Approche des problèmes sociaux ", dans Fernand Dumont, Simon Langlois et Yves Martin (dir.), Traité des problèmes sociaux, Ste-Foy, Presses de l'Université Laval, p. 1-22.

DURKHEIM, Émile (2004). Les règles de la méthode sociologique, Quadrige, $12^{e}$ édition, PUF, 176 p.

EKMAN, Paul, et Richard J. DAVIDSON (1994). The nature of émotion, fondamental questions, New York, Oxford University Press, $498 \mathrm{p}$.

ENRIQUEZ, Eugène (1992). L'organisation en analyse, Paris, PUF, 334 p.

EHRENBER G, Alain (1998). La fatigue d'être soi. Dépression et société, Odile Jacob, Paris, 414 p.

FARRUGIA, Francis (2010). "Le syndrome narratif : une inquiétante étrangeté ", SociologieS, Dossiers Émotions et sentiments, réalité et fiction, réf du 17 février 2011, http//sociologies.revues. org/ index 3152.html.

FARRUGIA, Francis (dir.) (2002). La connaissance sociologique, Paris, L'Harmattan, 218 p.

GARDIN,Jean-Claude (2001). « Modèles et Récits », dans Jean-Michel Berthelot (dir.), Épistémologie des sciences sociales, Paris, PUF, p. 407-450.

GIDDENS, Anthony (1984). La constitution de la société, Paris, PUF, 474 p.

GURVITCH, Georges (2007). «Théories de l'expérience intégrale de l'immédiat. Extrait de Morale théorique et science des mœurs. Leurs possibilités, leurs conditions ", SociologieS, réf. du 30 octobre 2010, http://sociologies.revues.org/index1333.html

LEBRETON, David (2004). Les passions ordinaires, Paris, Payot, 346 p.

MARTUCCELLI, Danilo (2006). Forgé par l'épreuve. L'individu dans la France contemporaine, Paris, Armand Colin, $478 \mathrm{p}$.

MEAD, Margaret (1973). Moeurs et sexualité en Océanie, Paris, Plon, 533 p.

PIAGET, Jean (2005). L'épistémologie génétique, Paris, PUF, 126 p.

REID, Wilfrid (2008). "Un nouveau regard sur la pulsion, le trauma et la méthode analytique.

Première partie : une théorie de la psyché ", Filigrane,Vol. 17, № 1, p. 68-94. 
RENAULT, Emmanuel (2008). Souffrances sociales : philosophie, psychologie et politique, Paris, Éditions La Découverte, $405 \mathrm{p}$.

RENAUD, Gilbert (2001). «Intervenir, une question de sens » dans Crise de société... recherche de sens, Actes du colloque, ACSM section Montréal, p. 95-115.

SÉVIGNY, Robert, (2009). "Sociologie clinique et schizophrénie en Chine post-maö̈ste : l'expérience de Lu Lu ", Sociologie et sociétés, Vol. 41, № 1, Presses de l'Université de Montréal, p. $125-158$.

SCHURMANS, Marie-Noelle (2010). « D'amour et de feu », SociologieS, réf. du 30 octobre 2010, $\mathrm{http} /$ /sociologies.revues.org/index3157.html.

SIMMEL, Georg (1999). Sociologie, étude des formes de la socialisation, Paris, PUF, 772 p.

SIMMEL, Georg, (1988). La tragédie de la culture et autres essais, Rivages, $254 \mathrm{p}$.

VYGOTSKY, Lev (1998). Théorie des émotions, étude historico psychologique, Paris l'Harmattan, 416 p. 